\title{
Claves para la creación entornos virtuales de aprendizaje de la asignatura Derecho Financiero I.
}

Irune Suberbiola Garbizu ${ }^{\mathrm{a}}$

aUniversidad del País Vasco-Euskal Herriko Unibertsitatea, irune.suberbiola@ehu.eus.

\begin{abstract}
The teaching of Financial and Tax Law at the University of the Basque Country is circumscribed to the environment of the Degree in Law and the Double Degree in Law-Business Administration, and it's limited in its content, the number of students and the physical and temporal space assigned to it. Likewise, these limitations constrain the teaching tools to be used, hindering the use of active methodologies that promote the significant learning of this discipline. New technologies, the creation of online contents and the establishment of virtual and blended environments are essential tools to address the search for new knowledge that transcends to more complex scenarios, those required in professional environments. The incorporation of $I C T$, Web 2.0 instruments and VLCs represent an effective resource to implement a deep learning of Tax Law, but, for this aim, some elements and conditions that facilitate it are required. Thus, although achieving meaningful learning depends "on the one who learns", it is up to the teacher to generate the appropriate climate and the strategies that motivate that learning to develop. This paper reflects on the virtual modality in our faceto-face teaching practice, proposing the design of materials and online activities that promote the significant learning of Financial and Tax Law.
\end{abstract}

Keywords: teaching technological resources, virtual environments, support for face-to-face teaching, meaningful learning, Financial and Tax Law.

\footnotetext{
Resumen

La enseñanza del Derecho Financiero y Tributario en la UPV-EHU se circunscribe al entorno del Grado en Derecho y del Doble Grado en Derecho-ADE, limitándose en cuanto a su contenido, al número de alumnos y al espacio fisico y temporal que se le asigna. Igualmente, estas limitaciones constriñen los instrumentos docentes a utilizar, dificultando el empleo de metodologías activas que impulsen el aprendizaje significativo de esta disciplina. Las nuevas tecnologías, la creación de contenidos online y el establecimiento de entornos virtuales y semipresenciales se erigen como herramientas fundamentales para abordar la búsqueda de nuevos conocimientos que trasciendan a escenarios más complejos, los requeridos en entornos profesionales. La incorporación de las TIC, los instrumentos Web 2.0 y las CVA suponen un recurso eficaz para implementar un
} 
aprendizaje profundo del Derecho Tributario, pero, para ello, se requieren elementos y condiciones que lo faciliten. Así, si bien lograr un aprendizaje significativo depende "del que aprende", corresponde al docente generar el clima adecuado y las estrategias que motiven que ese aprendizaje se desarrolle. Esta comunicación reflexiona sobre la modalidad virtual en nuestra práctica docente presencial, planteando el diseño de materiales y de actividades online que impulsen el aprendizaje significativo del Derecho Financiero y Tributario.

Palabras clave: recursos tecnológicos para la docencia, entornos virtuales, apoyo a la docencia presencial, aprendizaje significativo, Derecho Financiero y Tributario.

\section{Introducción}

El Derecho Financiero y Tributario está presente, de una forma $u$ otra, en todos los actos $u$ operaciones que realizan tanto las personas físicas como las jurídicas. Bajo determinadas circunstancias, todos los años la mayoría de las primeras han de presentar y liquidar el Impuesto sobre la Renta de las Personas Físicas (IRPF) por la renta obtenida en el ejercicio fiscal precedente. De igual modo, las sociedades mercantiles y otras personas jurídicas han de presentar la declaración-liquidación del impuesto que grava sus beneficios, el Impuesto sobre Sociedades (IS). Por otro lado, profesionales y empresarios han de repercutir el Impuesto sobre el Valor Añadido (IVA) y gestionar dicho tributo en relación con su actividad económica. Al mismo tiempo, y sin necesidad de que medie dicha actividad económica, periódicamente se cobran determinados impuestos que gravan la propiedad inmobiliaria (Impuesto sobre Bienes Inmuebles), o determinados servicios públicos prestados en relación con la misma, (como sería el caso de las tasas relacionadas con la gestión de residuos, las tasas de alcantarillado...) Ocasionalmente, a lo largo de nuestra vida, como cuando adquirimos bienes inmuebles de segunda mano, percibimos una herencia o una donación,... también se han de liquidar e ingresar los impuestos que gravan dichas adquisiciones. En resumen, vivimos rodeados de situaciones, hechos, y negocios de los que derivan consecuencias tributarias cuyo conocimiento es básico para cualquier jurista.

En este contexto, el objetivo del Derecho Financiero y Tributario es explicar al alumno el cauce jurídico por el que se relacionan la Administración Tributaria (estatal, autonómica, foral o local) y los contribuyentes, con ocasión de la puesta en marcha de los procedimientos de aplicación de los tributos (gestión, inspección o recaudación), la revisión de los mismos, así como de la posible imposición de sanciones por incumplimiento de su obligación de pago. En otras palabras, además de estudiar los principios generales que en el ámbito financiero-tributario se recogen en nuestro ordenamiento, se centra en los procedimientos comunes aplicables a los tributos que constituyen el sistema tributario español, cualquiera que sea el poder financiero del que emanan. Su estudio resulta en 
consecuencia imprescindible para cualquier profesional del ámbito jurídico o económico que necesite conocer las normas que entran en juego para regular las relaciones jurídicas que surgen entre contribuyentes y Administraciones tributarias, así como para calibrar el adecuado respeto a las normas legales por cualquiera de ambos sujetos.

La iniciativa propuesta se dirige principalmente a alumnos de la parte general (Derecho Financiero I) en el Grado en Derecho de la Universidad del País Vasco, siendo su finalidad facilitar a los participantes un entorno virtual donde abundar en aquellos conceptos que por cuestiones temporales resulta imposible examinar en el aula. De este modo, su estudio se plantea como un recurso adicional, un apoyo a la docencia presencial mediante una plataforma online para profundizar en aspectos no ahondados o poco trabajados en clase.

Históricamente la enseñanza del Derecho Financiero y Tributario y su acomodo a las nuevas tecnologías ha sufrido los avatares que tan gráficamente describen Area Moreira (2018) y Moreno González y Luchena Mozo (2014). Para hacer frente a estas situaciones, en la actualidad las plataformas suministradas por la Universidad del País Vasco-Euskal Herriko Unibertsitatea a través del servicio eCampus (aquel que se encarga de gestionar y desarrollar todos los aspectos relativos al aprendizaje y la enseñanza online, tanto en la formación reglada como en la formación continua) son cuatro; el gestor de aulas virtuales para titulaciones oficiales eGela (con 40.000 usuarios activos y 8.000 cursos), Egelapi, aulas virtuales para títulos propios y otras actividades (con 5.700 usuarios activos y 300 cursos), OpenCourseWare, con recursos abiertos desde 2008 para el aprendizaje para cerca de 320 cursos y convenios anuales, y Miríada, plataforma de cursos MOOC en español y portugués que cuenta con 12 cursos en las convocatorias anuales celebradas desde 2014 .

Precisamente la finalidad coadyuvante de la iniciativa presentada, el ser un proyecto que se plantea en términos de apoyo a la docencia presencial, el que estemos hablando de una suerte de b-learning, determina que, entre las plataformas facilitadas por eCampus, la plataforma a utilizar para para la creación de un curso de apoyo a la docencia presencial en un entorno virtual de aprendizaje de nuestra disciplina sea eGela-Moodle, y ello para que pueda ser un complemento a los alumnos matriculados en la asignatura Derecho Financiero $\mathrm{I}$, sin necesidad de que cumplan o se registren en ninguna otra adicional a la que les es propia por el mero hecho de estar matriculados como alumnos. En este sentido, la plataforma seleccionada, como $\mathrm{LCMS}^{1}$, permite la utilización recursos tradicionales (como repositorio de contenidos, ficheros libros, páginas, enlaces web...), la interacción entre alumn@-profesor@ (mediante la entrega y evaluación de distintas tareas, cuestionarios...), entre el propio alumnado (a modo de red social para la creación de contenidos colaborativos, para la comunicación síncrona y asíncrona entre los mismos gracias a foros, glosarios, talleres, wikis...) o a la combinación de todos los anteriores ${ }^{2}$.

\footnotetext{
${ }^{1}$ Learning Content Management System

${ }^{2}$ Ver al respect BAUTISTA, G. ; BORGES, F. ; FORÉS, A. (2006) "Didáctica universitaria en entornos virtuales de enseñanzaaprendizaje". Narcea: Madrid.
} 


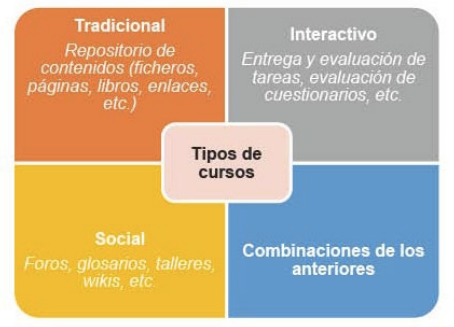

Fig. 1. Características plataforma virtual utilizada

Partiendo de las características propias de la plataforma eGela-Moodle, esta comunicación recoge los objetivos, el desarrollo de la innovación y los resultados obtenidos en la creación de un entorno virtual en apoyo al aprendizaje del Derecho Financiero y Tributario, en concreto de su parte general, la asignatura Derecho Financiero I.

\section{Objetivos}

La innovación introducida pretende dotar de cierta coherencia a los contenidos, herramientas y programación docente de la asignatura Derecho Financiero I, como elemento coadyuvante al desarrollo de su docencia presencial. Como mencionábamos, las características temporales, la carga lectiva asignada y la composición de los grupos donde se imparte la asignatura en el Grado en Derecho imposibilita la óptima consecución de los objetivos de aprendizaje y competencias a desarrollar en la misma, lo que, sin duda, convierte al entorno virtual eGela-Moodle en la herramienta principal para su apropiada culminación.

Con esta finalidad se plantea el diseño de unas competencias y resultados de aprendizaje, contenidos, metodología, plan de trabajo y actividades evaluadas, cronograma y sistema de evaluación adaptados al entorno virtual que sirvan como herramientas auxiliares de las clases presenciales, de forma que el alumno haya trabajado previamente, a modo de flipped classroom, los contenidos propuestos. Del mismo modo, la iniciativa se plantea como un instrumento que impulsa el aprendizaje significativo del alumnado de forma que los conocimientos previos (incluso los adquiridos fuera del entorno académico) se erijan como bloques en la construcción paulatina de la comprensión de esta disciplina. La adquisición de nuevos conocimientos se hace forma relacional, imbricando una nueva información con aquella que ya se posee, lo que permite reajustar y reconstruir ambas informaciones dotándoles un de un nuevo alcance que, a su vez, permite a futuro la recepción y afianzamiento de nuevos conocimientos.

\section{Desarrollo de la innovación}

\subsection{Competencias y resultados de aprendizaje propuestos}

Las competencias y resultados de aprendizaje propuestos para el entorno virtual aúnan las que son propias de la asignatura con las inherentes a las destrezas necesarias para un 
adecuado desenvolvimiento en este tipo de plataformas. Pasamos a resumirlas en las siguientes tablas sinópticas.

Tabla 1. Competencias del curso

\begin{tabular}{|c|c|c|}
\hline \multicolumn{3}{|c|}{ COMPETENCIAS DEL CURSO } \\
\hline $\mathbf{N}^{\circ}$ & DESCRIPCIÓN & TIPO \\
\hline $\mathrm{Cl}$ & Capacidad de reflexión y habilidad para relacionar ideas elaborando argumentaciones juridicas. & Especifica \\
\hline $\mathrm{C} 2$ & Tener una visión transversal de los efectos juridicos de una misma realidad & Específica \\
\hline $\mathrm{C} 3$ & $\begin{array}{l}\text { Desarrollar una aptitud critica tanto en la búsqueda de información como en los objetivos que } \\
\text { fundamentan las normas y en la confección de conclusiones y propuestas alternativas }\end{array}$ & Específica \\
\hline $\mathrm{C} 4$ & Desarrollar una visión unitaria e interdisciplinar del derecho & Especifica \\
\hline $\mathrm{C} 5$ & $\begin{array}{l}\text { Gestionar el proceso de aprendizaje continuado y autónomo, de modo que se obtenga una } \\
\text { formación integral y coherente que permita poder afrontar con éxito estudios posteriores de } \\
\text { especialización o reciclaje }\end{array}$ & Transversal \\
\hline C6 & $\begin{array}{l}\text { Aplicar adecuadamente los conocimientos y técnicas adquiridos a lo largo de los estudios de } \\
\text { grado en orden a la correcta resolución de los problemas jurídicos, adoptando decisiones de } \\
\text { manera creativa y afrontando situaciones diversas, apoyado en una suficiente capacidad de } \\
\text { organización, trabajo en pequeños grupos y planificación }\end{array}$ & Transversal \\
\hline $\mathrm{C} 7$ & $\begin{array}{l}\text { Adoptar decisiones de manera creativa, afrontando situaciones diversas, apoyándose en una } \\
\text { suficiente capacidad de organización, trabajo en pequeños grupos y planificación. }\end{array}$ & Transversal \\
\hline $\mathrm{C} 8$ & $\begin{array}{l}\text { Capacidad de reunir e interpretar datos relevantes para emitir juicios que incluyan una reflexión } \\
\text { sobre temas relevantes de indole social, cientifica o e etica }\end{array}$ & Transversal \\
\hline
\end{tabular}

Fuente: elaboración propia

Del mismo modo, el curso persigue una serie de objetivos de aprendizaje que, a su vez, pueden distinguirse entre los que son propios o específicos de la asignatura, y aquellos otros que lo son pero de una manera más transversal por ser comunes con el resto de materias que componen el Grado. En el siguiente cuadro distinguimos 12 objetivos de aprendizaje diferenciando entre objetivos específicos y transversales. 
Tabla 2. Objetivos de aprendizaje

\begin{tabular}{|c|c|c|}
\hline \multicolumn{3}{|c|}{ OBJETIVOS DE APRENDIZAJE DEL CURSO } \\
\hline $\mathbf{N}^{\circ}$ & DESCRIPCIÓN & TIPO \\
\hline OA1 & Identificar los distintos entes con poder tributario dentro de nuestro ordenamiento & Especifico \\
\hline $\mathrm{OA2}$ & Identificar los principios financieros y tributarios contenidos en nuestro ordenamiento. & Especifico \\
\hline $\mathbf{O A 3}$ & Identificar el concepto, clase y estructura de los tributos. & Especifico \\
\hline OA4 & $\begin{array}{l}\text { Identificar los elementos objetivos, subjetivos, espaciales, temporales y cuantitativos } \\
\text { derivados de la relación juridico-tributaria. }\end{array}$ & Especifico \\
\hline OA5 & Distinguir entre los diferentes procedimientos de aplicación de los tributos. & Especifico \\
\hline OA6 & $\begin{array}{l}\text { Identificar el alcance de los ilicitos tributarios y su repercusión en el ámbito sancionador de la } \\
\text { Administración Tributaria y el Derecho Penal }\end{array}$ & Especifico \\
\hline OA7 & Distinguir los distintos procedimientos de revisión de los tributos & Especifico \\
\hline OA8 & Desarrollar una argumentación jurídica lógica. & Transversal \\
\hline OA9 & $\begin{array}{l}\text { Adquirir habilidades para analizar problemas jurídicos complejos y búsqueda de soluciones } \\
\text { adecuadas conforme a la normativa aplicable }\end{array}$ & Transversal \\
\hline OA10 & $\begin{array}{l}\text { Adquisición de habilidades (orales y escritas) para la exposición de trabajos, con } \\
\text { profesionalidad y con la oportuna sensibilidad que requiere la materia. }\end{array}$ & Transversal \\
\hline OA11 & $\begin{array}{l}\text { Adquisición de destrezas para la realización de casos prácticos, de manera que se demuestre } \\
\text { una suficiente habilidad para enfrentarse a problemas cotidianos de manera constructiva }\end{array}$ & Transversal \\
\hline OA12 & $\begin{array}{l}\text { Realización de trabajos de profundización y síntesis a partir de búsquedas en las fuentes } \\
\text { bibliográficas e informáticas fundamentales relacionadas con la materia }\end{array}$ & Transversal \\
\hline
\end{tabular}

Fuente: elaboración propia

\subsection{Contenidos del curso}

Los contenidos del curso se estructuran en 7 Módulos temáticos, más un modulo 0 o introductorio que se presenta en el aula presencial, que incluyen los siguientes apartados:

\section{Módulo 0.- Presentación de la unidad Formativa online.}

- Presentación de la asignatura, de los recursos, materiales y actividades a realizar.

- Presentación de las distintas herramientas con las que contará el curso: foro; tutorías virtuales...

\section{Módulo 1.- Aspectos básicos del Derecho Financiero}

○ Derecho Financiero y Tributario.

- Entes con poder tributario.

- Fuentes del Derecho Tributario. 
Módulo 2.- El tributo

- Principios del Ordenamiento Tributario.

- Concepto, clases y finalidad de los tributos.

Módulo 3.- La relación jurídico tributaria.

- La relación jurídico tributaria

- Elementos subjetivos de las obligaciones tributarias

- Elementos de cuantificación de las obligaciones tributarias

- Deuda tributaria y extinción de las obligaciones tributarias

$>$ Módulo 4.- Procedimientos de aplicación de los tributos

- Aspectos comunes.

- Procedimientos de gestión.

- Procedimientos de inspección.

- Procedimientos de recaudación.

$>$ Módulo 5.- Ilícitos tributarios y procedimiento sancionador.

- Ilícitos tributarios.

- Procedimiento sancionador.

$>$ Módulo 6.- Recursos y reclamaciones contra la Hacienda.

- Procedimientos especiales de revisión de los tributos.

- Recurso de Reposición.

- Reclamación Económico Administrativa.

$>$ Módulo 7.- Integración práctica de los módulos anteriores.

\subsection{Metodología de trabajo}

Para que el curso se erija como un instrumento que coadyuve al desarrollo de las clases presenciales es necesaria la implantación de una perspectiva con una clara orientación teórico-práctica en la que los objetivos de aprendizaje y competencias pretendidas se adquieren a través del estudio razonado de todas las unidades didácticas propuestas en la plataforma, de modo que guían al estudiante en el estudio del material didáctico básico de la asignatura, así como del material didáctico complementario que se ponga a disposición. Junto con el material propuesto, se requiere que los estudiantes realicen una serie de Actividades de Aprendizaje (AA), Actividades de Evaluación Continua (AEC) y Controles recogidos en el plan de trabajo y actividades evaluadas y definidos en el sistema de evaluación ${ }^{3}$.

Tras una primera sesión bautizada como "módulo cero", en la que los alumnos serán informados de los recursos, materiales y actividades a realizar, así como de las distintas herramientas con las que contará el curso para su desarrollo, en el transcurso de la unidad formativa, las dudas conceptuales que puedan surgir tras el estudio razonado de los materiales colgados en los respectivos módulos deben plantearse en el foro de tutorías activado en el módulo cero de la plataforma eGela. Este foro general, además de ser el canal de comunicación prioritario con el profesor y con los compañeros del aula, pretende erigirse como herramienta primordial para el intercambio de conocimientos. En este sentido, las dudas que no queden resueltas en los glosarios o foros correspondientes, podrán

\footnotetext{
${ }^{3}$ Ver al respecto PRIETO, S. (2018) “Aprendizaje y tecnologías de la información y la comunicación”. Ed: CEF. Madrid, p. 67.
} 
ser atendidas telefónicamente en el horario de tutorías de la profesora (pertinentemente publicado en el módulo cero), y, si fuera necesario en las clases presenciales, pues, recordemos, la unidad formativa se plantea como una plataforma de ayuda a la docencia presencial. Igualmente, los alumnos podrán contar con la posibilidad de realizar tutorías virtuales con la profesora de la asignatura en la sala de video conexión (Blackboard Collaborate) o utilizar esta última para reunirse con otros alumnos para la elaboración de determinados trabajos colaborativos.

Los recursos a utilizar en el desarrollo de las diferentes actividades pueden ser de distinta índole en función del módulo que se vaya a trabajar, en cualquier caso podrían resumirse en los siguientes ${ }^{4}$.

- Vídeos resumen del contenido teórico de cada uno de los módulos.

- Documentos y archivos de diversa índole como textos legales, académicos, periodísticos sentencias, ...

- Consultas para realizar una encuesta rápida con el fin de que los alumnos reflexionen sobre un tema, para comprobar rápidamente que han entendido un punto en concreto...

- Cuestionarios con preguntas tipo opción múltiple, verdadero/falso, coincidencia, respuesta corta y respuesta numérica, y que pueden usarse para hacer exámenes del curso, mini test para tareas de lectura o al final de un tema a modo de one minute paper, controles de autoevaluación que nos faciliten información inmediata sobre el rendimiento de los alumnos...

- Glosario, para que los alumnos creen de forma colaborativa, a modo de diccionario, de términos y conceptos especialmente significativos para la materia.

- $\quad$ Tarea, que puede consistir en una AEC o una AA que luego la docente revisará, valorará, calificará y a la que podrá dar retroalimentación, y cuyo contenido digital puede ser diverso (documentos de texto, hojas de cálculo, imágenes, audio, vídeos...). Alternativamente la tarea podrá revisarse por pares entre los estudiantes de forma que se fomente el aprendizaje colaborativo y el sentido crítico sobre el contenido de la materia.

El tiempo destinado al estudio de cada unidad didáctica en particular y al curso en general es sumamente importante ${ }^{5}$. La exigencia de una dedicación excesiva podría desembocar en el abandono masivo de la asignatura, y, un tiempo demasiado limitado en el fracaso de los objetivos pretendidos. Teniendo en cuenta lo anterior, se estima que la lectura y comprensión de los contenidos teóricos abarcados en los distintos módulos ocupará en total unas 15 horas, mientras la realización de las distintas actividades evaluables, es decir, las

\footnotetext{
${ }^{4}$ Pese a que en un principio, en estadios anteriores de entornos virtuales se aconsejaba la utilización de $\underline{\text { Wikis, }}$, para que los alumnoseditaran, añadieran...una colección de páginas web y generean apuntes de clase colaborativamente el actual desarrollo tecnológico, la existencia de marcadores sociales fuera de moodle, como Diigo, Digg o Scoop.it hacen preferible la utilización de estos últimos y su integración y normalizaciónen la vida cotidiana de nuestros estudiantes

${ }^{5}$ BORGES, F. (2005). "La frustración del estudiante en línea. Causas y acciones preventivas". Digithum [artículo en línea]. UOC. $\mathrm{N}^{\mathrm{o}}$ 7, http://www.uoc.edu/digithum/7/dt/esp/borges.pdf [Consulta: 19 de febrero de 2019]
} 
Actividades de Evaluación Continua (AEC), las Actividades de Aprendizaje (AA) y la realización de los Controles conllevará aproximadamente otras 22 horas de dedicación a lo largo de las 11 semanas que durará el curso. Se considera que el empleo de unas 10 horas por parte del alumno a la hora de preparar el examen final presencial será suficiente para consolidar los conocimientos adquiridos durante el transcurso de la asignatura mediante la realización de las distintas tareas y lecturas requeridas. Finalmente, antes de terminar el curso, y para poder acceder a participar en examen presencial, cada participante deberá haber obtenido al menos el 50\% de la nota correspondiente al conjunto de actividades (AA, AEC y controles) sugeridas en la unidad formativa, es decir, deberá alcanzar, al menos un 2,5 en la nota total de estas actividades.

\subsection{Plan de trabajo $y$ actividades evaluadas}

El plan de trabajo que se establece en torno a los 7 Módulos, así como la actividad evaluada en cada uno de ellos es el siguiente:

Tabla 3. Módulo 1

\begin{tabular}{|c|c|c|c|}
\hline \multicolumn{4}{|c|}{ MÓDULO 1: Aspectos básicos del Derecho Financiero } \\
\hline $\begin{array}{l}\text { Objetivo de } \\
\text { aprendizaje }\end{array}$ & $\begin{array}{l}\text { Contenidos } \\
\text { temáticos }\end{array}$ & Materiales a utilizar & Actividades a realizar \\
\hline $\begin{array}{c}\text { OA1 } \\
\text { Identificar los distintos } \\
\text { entes con poder } \\
\text { tributario dentro de } \\
\text { nuestro ordenamiento. } \\
\text { Subsidiariamente } \\
\text { analizar la relación } \\
\text { entre poder financiero y } \\
\text { las fuentes del Derecho } \\
\text { Financiero y Tributario. }\end{array}$ & $\begin{array}{ll}- & \text { Derecho } \\
& \text { Financiero y } \\
\text { Tributario. } \\
\text { - } & \text { Entes con } \\
\text { poder } \\
\text { tributario. } \\
\text { - } & \text { Fuentes del } \\
\text { Derecho } \\
\text { Tributario. }\end{array}$ & $\begin{array}{l}\text { - Video de presentación } \\
\text { - Archivo normativa en } \\
\text { vigor: Constitución, TFUE, } \\
\text { Concierto Económico. } \\
\text { - Archivo textos académicos } \\
\text { - Links a artículos } \\
\text { periodisticos. }\end{array}$ & $\begin{array}{l}\text { - Cuestionario previo. } \\
\text { - Tarea AA: Mapa conceptual entes con poder tributario. } \\
\text { - Glosario } \\
\text { - Foro: comentario sobre alguna noticia relacionada con la materia. } \\
\text { - Cuestionario a realizar a la finalización del módulo para la } \\
\text { autoevaluación del alumno. }\end{array}$ \\
\hline \multicolumn{4}{|c|}{ Actividad evaluada 1: Mapa conceptual sobre los distintos entes con poder tributario en nuestro ordenamiento } \\
\hline Tipo de actividad & \multicolumn{3}{|l|}{ AAl } \\
\hline Descripción & \multicolumn{3}{|c|}{$\begin{array}{l}\text { Desarrollo de un mapa conceptual en el que se recojan los aspectos más significativos de los distintos entes con poder tributario en } \\
\text { nuestro ordenamiento (UE, Estado, Comunidades Autónomas de Régimen Común, Territorios Forales, Ayuntamientos), con } \\
\text { indicación de las potestades conferidas a cada uno, ámbito de actuación, tributos sobre los que tienen competencias... }\end{array}$} \\
\hline Objetivo & \multicolumn{3}{|c|}{$\begin{array}{l}\text { OAl Identificar los distintos entes con poder tributario dentro de nuestro ordenamiento, la relación entre ellos y la trascendencia que } \\
\text { este hecho tiene para los ciudadanos. }\end{array}$} \\
\hline Materiales necesarios & \multicolumn{3}{|c|}{$\begin{array}{l}\text { Además de los propios del módulo como herramienta es indiferente el la herramienta utilizada para la concepción del mapa } \\
\text { conceptual (puede ser CmapTools o cualquier otra). }\end{array}$} \\
\hline
\end{tabular}

Tabla 4. Módulo 2

\begin{tabular}{|c|c|c|c|}
\hline \multicolumn{4}{|c|}{ MÓDULO 2: El tributo } \\
\hline $\begin{array}{l}\text { Objetivo de } \\
\text { aprendizaje }\end{array}$ & $\begin{array}{l}\text { Contenidos } \\
\text { temáticos }\end{array}$ & Materiales a utilizar & Actividades a realizar \\
\hline $\begin{array}{c}\text { OA } 2 \\
\text { Identificar los } \\
\text { Principios financieros y } \\
\text { tributarios contenidos } \\
\text { en nuestro } \\
\text { ordenamiento. } \\
\text { OA } 3 \\
\text { Identificar el concepto, } \\
\text { clase y estructura de los } \\
\text { tributos }\end{array}$ & $\begin{array}{l}\text { - Principios del } \\
\text { Ordenamiento } \\
\text { Tributario } \\
\text { - Concepto, clases } \\
\text { y finalidad de } \\
\text { los tributos }\end{array}$ & $\begin{array}{l}\text { - Video de presentación } \\
\text { - Archivo normativa en } \\
\text { vigor: LGT, NFGT. } \\
\text { - Archivo textos } \\
\text { académicos } \\
\text { - Links a articulos } \\
\text { periodisticos }\end{array}$ & $\begin{array}{l}\text { - Cuestionario previo. } \\
\text { - Tarea AECl: Análisis de un determinado impuesto a la luz de los principios de justicia } \\
\text { tributaria material. } \\
\text { - Glosario } \\
\text { - Foro: comentario sobre alguna noticia relacionada con la materia. } \\
\text { - Cuestionario a realizar a la finalización del módulo para la autoevaluación del alumno. }\end{array}$ \\
\hline \multicolumn{4}{|c|}{ Actividad evaluada 2: Análisis de los tributos desde la perspectiva de los principios contenidos en el artículo 31 C.E } \\
\hline $\begin{array}{l}\text { Tipo de actividad } \\
\text { Descripción } \\
\text { Objetivo }\end{array}$ & $\begin{array}{l}\text { AEC } 1 \\
\text { Análisis de un determ } \\
\text { OA2 Revelar que seg } \\
\text { impuestos sino que de } \\
\text { Generar una visión cr }\end{array}$ & $\begin{array}{l}\text { lado impuesto a la luz de lo } \\
\text { la jurisprudencia del } \mathrm{TC} \text {, } \\
\text { ende del lugar que éstos oc } \\
\text { ca sobre este hecho. }\end{array}$ & $\begin{array}{l}\text { ncipios de justicia tributaria material } \\
\text { scendencia de los principios tributarios de justicia material no es homogénea en todos los } \\
\text { en la estructura general impositiva. }\end{array}$ \\
\hline Materiales necesarios & Textos legales, doctri & y jurisprudencia. & \\
\hline
\end{tabular}


Tabla 5. Módulo 3

\begin{tabular}{|c|c|c|c|}
\hline \multicolumn{4}{|c|}{ MÓDULO 3: La relación jurídico tributaria } \\
\hline $\begin{array}{l}\text { Objetivo de } \\
\text { aprendizaje }\end{array}$ & $\begin{array}{c}\text { Contenidos } \\
\text { temáticos }\end{array}$ & Materiales a utilizar & Actividades a realizar \\
\hline $\begin{array}{c}\text { OA 4 } \\
\text { Identificar los } \\
\text { elementos objetivos, } \\
\text { subjetivos, espaciales, } \\
\text { temporales y } \\
\text { cuantitativos derivados } \\
\text { de la relación juridico- } \\
\text { tributaria }\end{array}$ & $\begin{array}{l}\text { - La relación } \\
\text { jurídico } \\
\text { tributaria } \\
\text { - Elementos } \\
\text { subjetivos de las } \\
\text { obligaciones } \\
\text { tributarias } \\
\text { - Elementos de } \\
\text { cuantificación } \\
\text { de las } \\
\text { obligaciones } \\
\text { tributarias } \\
\text { - Deuda tributaria } \\
\text { y extinción de } \\
\text { las obligaciones } \\
\text { tributarias } \\
\end{array}$ & $\begin{array}{l}\text { - Vídeo de presentación. } \\
\text { - Apuntes/presentación } \\
\text { del docente. } \\
\text { - Archivo normativa en } \\
\text { vigor: LGT, NFGT. } \\
\text { - Archivo textos } \\
\text { académicos } \\
\text { - Links a artículos } \\
\text { periodísticos }\end{array}$ & $\begin{array}{l}\text { - Cuestionario previo. } \\
\text { - Tarea AA2: Creación de un tributo con finalidad extrafiscal. } \\
\text { - Glosario } \\
\text { - Foro: comentario sobre alguna noticia relacionada con la materia. } \\
\text { - Cuestionario a realizar a la finalización del módulo para la autoevaluación del } \\
\text { alumno. }\end{array}$ \\
\hline \multicolumn{4}{|c|}{ Actividad evaluada 3: Creación de un tributo con finalidad extrafiscal } \\
\hline Tipo de actividad & \multicolumn{3}{|l|}{ AA2 } \\
\hline Descripción & \multicolumn{3}{|c|}{$\begin{array}{l}\text { Redacción del articulado de un tributo que recoja la estructura de liquidación general de un impuesto atendiendo a los elementos que } \\
\text { componen la relación jurídico tributaria, los principios tributarios de justicia material así como los aspectos más esenciales del concepto. } \\
\text { Los alumnos también deben presentar un póster/infografía con la estructura del tributo. }\end{array}$} \\
\hline Objetivo & \multicolumn{3}{|c|}{$\begin{array}{l}\text { OA 2: Identificar los principios financieros y tributarios contenidos en nuestro ordenamiento. } \\
\text { OA 3: Identificar el concepto, clase y estructura de los tributos. } \\
\text { OA 4: Identificar los elementos objetivos, subjetivos, espaciales, temporales y cuantitativos derivados de la relación jurídico-tributaria. } \\
\text { OA 10: Adquisición de habilidades (orales y escritas) para la exposición de trabajos, con profesionalidad y con la oportuna sensibilidad } \\
\text { que requiere la materia. }\end{array}$} \\
\hline Materiales necesarios & \multicolumn{3}{|c|}{$\begin{array}{l}\text { Además de los propios del módulo como herramienta un procesador de textos. Es indiferente el la herramienta utilizada para la } \\
\text { concepción del póster/infografia (Easel.ly, Genial.ly; Piktochart... o cualquier otra). }\end{array}$} \\
\hline
\end{tabular}

Actividad evaluada 4: Control módulos 1-3

Tipo de actividad Control

Descripción Control sobre el contenido de los módulos 1-3

Objetivo OA 1 Identificar los distintos entes con poder tributario dentro de nuestro ordenamiento, la relación entre ellos y la trascendencia que este hecho tiene para los ciudadanos

$O A$ 2: Identificar los principios financieros y tributarios contenidos en nuestro ordenamiento.

OA 3: Identificar el concepto, clase y estructura de los tributos.

OA 4: Identificar los elementos objetivos, subjetivos, espaciales, temporales y cuantitativos derivados de la relación jurídico-tributaria.

Materiales necesarios Además de los propios del módulo como herramienta el cuestionario de la plataforma; encuesta google drive; socrative...

\section{Tabla 6. Módulo 4}

\begin{tabular}{|c|c|c|c|}
\hline \multicolumn{4}{|c|}{ MóDULO 4: Procedimientos de aplicación de los tributos } \\
\hline $\begin{array}{l}\text { Objetivo de } \\
\text { aprendizaje }\end{array}$ & $\begin{array}{l}\text { Contenidos } \\
\text { temáticos }\end{array}$ & Materiales a utilizar & Actividades a realizar \\
\hline $\begin{array}{l}\text { OA } 5 \\
\text { Distinguir entre los } \\
\text { diferentes } \\
\text { procedimientos de } \\
\text { aplicación de los } \\
\text { tributos }\end{array}$ & $\begin{array}{l}\text { - Aspectos } \\
\text { comunes. } \\
\text { - Procedimientos } \\
\text { de gestión. } \\
\text { - Procedimientos } \\
\text { de inspección. } \\
\text { - Procedimientos } \\
\text { de recaudación. }\end{array}$ & $\begin{array}{l}\text { - Video de presentación. } \\
\text { - Apuntes / presentación } \\
\text { del docente. } \\
\text { - Archivo normativa en } \\
\text { vigor: LGT, NFGT. } \\
\text { Reglamentos de } \\
\text { desarrollo. } \\
\text { - Archivo textos } \\
\text { académicos }\end{array}$ & $\begin{array}{l}\text { - Cuestionario previo. } \\
\text { - Tarea AEC2: resolución de un caso práctico relacionado con uno de los } \\
\text { procedimientos de aplicación de los tributos. } \\
\text { - Glosario } \\
\text { - Foro: comentario sobre alguna noticia relacionada con la materia. } \\
\text { - Cuestionario a la finalización del módulo para la autoevaluación. }\end{array}$ \\
\hline \multicolumn{4}{|c|}{ Actividad evaluada 5: Resolución de caso práctico } \\
\hline Tipo de actividad & \multicolumn{3}{|l|}{$\mathrm{AEC} 2$} \\
\hline Descripción & \multicolumn{3}{|c|}{ Resolución de un caso práctico relacionado con uno de los procedimientos de aplicación de los tributos a elección del alumno. } \\
\hline Objetivo & \multicolumn{3}{|c|}{$\begin{array}{l}\text { OA 5: Distinguir entre los diferentes procedimientos de aplicación de los tributos. } \\
\text { OA 8: Desarrollar una argumentación juridica lógica. } \\
\text { OA 9: Adquirir habilidades para analizar problemas jurídicos complejos y búsqueda de soluciones adecuadas conforme a la normativa } \\
\text { aplicable } \\
\text { OA 11: Adquisición de destrezas para la realización de casos prácticos, de manera que se demuestre una suficiente habilidad para enfrentarse a } \\
\text { problemas cotidianos de manera constructiva. }\end{array}$} \\
\hline Materiales necesarios & \multicolumn{3}{|c|}{ Además de los propios del módulo como herramienta un procesador de textos. } \\
\hline
\end{tabular}


Tabla 7. Módulo 5.

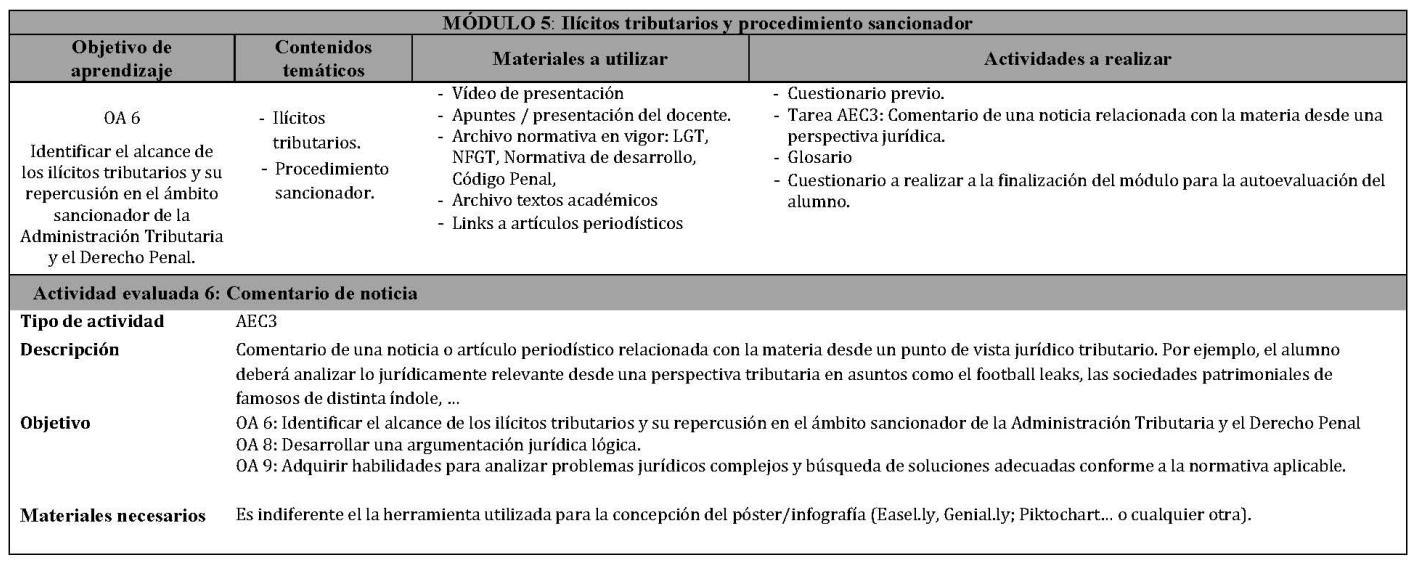

Tabla 8. Módulo 6

\begin{tabular}{|c|c|c|c|}
\hline \multicolumn{4}{|c|}{ MóDULO 6: Recursos y reclamaciones contra la Hacienda } \\
\hline $\begin{array}{l}\text { Objetivo de } \\
\text { aprendizaje }\end{array}$ & Contenidos temáticos & Materiales a utilizar & Actividades a realizar \\
\hline $\begin{array}{l}\text { OA } 7 \\
\text { Distinguir los distintos } \\
\text { procedimientos de } \\
\text { revisión de los tributos }\end{array}$ & $\begin{array}{l}\text { - Procedimientos } \\
\text { especiales de } \\
\text { revisión de los } \\
\text { tributos. } \\
\text { - Recurso de } \\
\text { Reposición. } \\
\text { - Reclamación } \\
\text { Económico } \\
\text { Administrativa. }\end{array}$ & $\begin{array}{l}\text { - Video de presentación } \\
\text { - Apuntes / presentación del } \\
\text { docente. } \\
\text { - Archivo normativa en vigor: } \\
\text { LGT, NFGT, Normativa de } \\
\text { desarrollo. } \\
\text { - Archivo textos académicos }\end{array}$ & $\begin{array}{l}\text { - Cuestionario previo. } \\
\text { - Tarea AA3: línea del tiempo. } \\
\text { - Glosario } \\
\text { - Foro: comentario sobre ADR tributario } \\
\text { - Cuestionario a realizar a la finalización del módulo para la autoevaluación } \\
\text { del alumno. }\end{array}$ \\
\hline \multicolumn{4}{|c|}{ Actividad evaluada 7: Línea del tiempo } \\
\hline Tipo de actividad & \multicolumn{3}{|l|}{ AA3 } \\
\hline Descripción & \multicolumn{3}{|c|}{$\begin{array}{l}\text { Desarrollar un cronograma con las distintas posibilidades/alternativas que confluyen por un lado en los procedimientos de aplicación de los } \\
\text { tributos y en el procedimiento sancionador y las opciones de revisión de las resoluciones administrativas adoptadas en estos procedimientos } \\
\text { mediante los diferentes recursos y reclamaciones que existen contra la Hacienda. }\end{array}$} \\
\hline Objetivo & \multicolumn{3}{|c|}{$\begin{array}{l}\text { A 5: Distinguir entre los diferentes procedimientos de aplicación de los tributos. } \\
\text { OA 6: Identificar el alcance de los ilícitos tributarios y su repercusión en el ámbito sancionador de la Administración Tributaria y el Derecho } \\
\text { Penal. } \\
\text { OA7 } \\
\text { OA 9: Adquirir habilidades para analizar problemas juridicos complejos y búsqueda de soluciones adecuadas conforme a la normativa } \\
\text { aplicable }\end{array}$} \\
\hline Materiales necesarios & \multicolumn{3}{|c|}{ Además de los propios del módulo como es indiferente el la herramienta utilizada para la concepción de la línea del tiempo. } \\
\hline
\end{tabular}


Tabla 9. Módulo 7

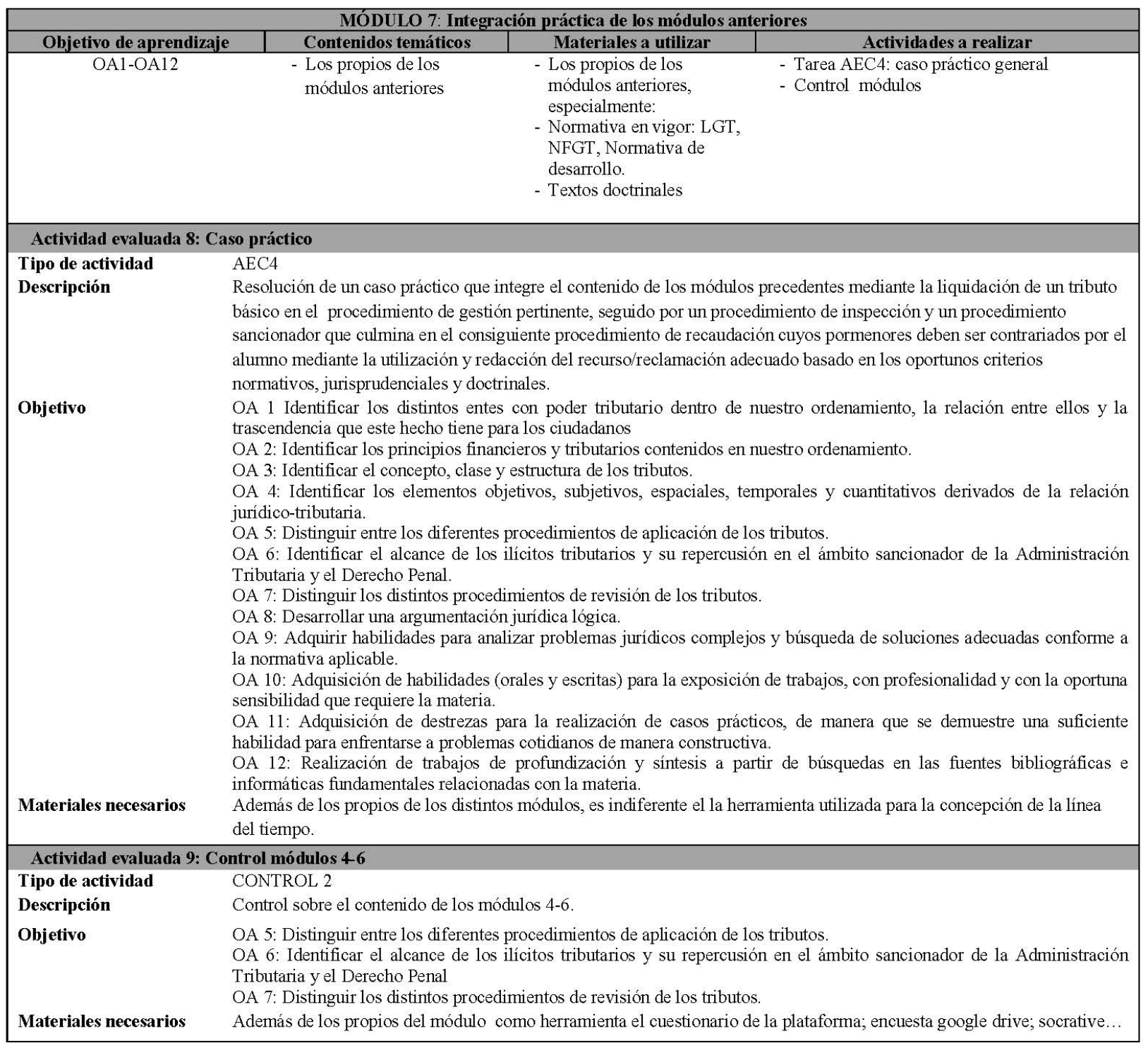

\subsection{Evaluación}

Según comentábamos en apartados precedentes, la evaluación de estas unidades formativas online contempla la realización de diferentes tipos de actividades de evaluación y aprendizaje cuyas fechas de realización se encuentran recogidas en el apartado siguiente (cronograma). La evaluación formativa, que comprende Actividades de Aprendizaje (AA), Actividades de Evaluación Continua (AEC), y los Controles, supone un $50 \%$ de la nota final. Por su parte, el examen final presencial, que valora la adquisición de los 
conocimientos por parte del estudiante tiene un peso del $50 \%$ restante en la nota final de la asignatura.

Además, para presentarse al examen final presencial y poder superar el curso es requisito indispensable haber obtenido al menos el $50 \%$ de la nota correspondiente al conjunto total de actividades del aula virtual, es decir, haber alcanzado al menos un 2,5 en la nota total de las actividades AA, AEC y Controles.

Tabla 10. Evaluación 1

\begin{tabular}{|l|c|}
\hline \multicolumn{1}{|c|}{ TIP0 DE ACTIVIDAD } & \% DE LA NOTA FINAL \\
\hline AA & $20 \%$ \\
\hline AEC & $20 \%$ \\
\hline Controles & $10 \%$ \\
\hline Examen final presencial & $50 \%$ \\
\hline Total & $100 \%$ \\
\hline
\end{tabular}

Cada una de las actividades evaluadas en el aula virtual así como el examen final presencial deberían ser calificadas con el siguiente porcentaje:

Tabla 11. Porcentajes de las actividades evaluadas

\begin{tabular}{|c|c|c|c|c|c|c|c|c|c|c|c|}
\hline $\begin{array}{c}\text { Activi } \\
\text { dad }\end{array}$ & AA1 & AA2 & AA3 & AEC1 & AEC2 & AEC3 & AEC4 & C1 & C2 & examen & \multicolumn{2}{|c|}{} \\
\hline$\%$ & 6,66 & 6,66 & 6,66 & 3,00 & 7,00 & 2,00 & 8,00 & 5,00 & 5,00 & 50 & $100 \%$ \\
\hline
\end{tabular}

Las actividades de aprendizaje del aula no realizadas obtendrán puntuación igual a cero.

Por cuestiones de agilidad, se recomienda que el examen final presencial de la unidad formativa se compone de dos partes, una primera parte tipo test y una segunda parte de tres preguntas de desarrollo.

- El Test de opción multiple debería ser rápido, por ejemplo, constando de diez preguntas con varias opciones de respuesta cada una, donde una única solución es válida. La nota obtenida en este apartado constituye el $40 \%$ de la calificación global del examen presencial. Cada pregunta acertada suma 0,4 puntos y las preguntas erróneas penalizan la puntuación.

- En cuanto a las preguntas de desarrollo se propondrán tres temas o preguntas sobre el temario de la asignatura. La nota obtenida en este apartado constituye el $60 \%$ de la calificación global del examen presencial.

La calificación global obtenida en el examen presencial, una vez sumadas las de los dos apartados previamente descritos, representa un $50 \%$ de la nota final de la asignatura. El examen final presencial deberá superarse, con al menos un cinco (5.0), para poder promediar con las calificaciones obtenidas en las AA, AEC y Controles. En este sentido, aunque un alumno apruebe el examen presencial, si la calificación resultante de sumar la obtenida en dicha prueba y las notas procedentes del resto de apartados (AEC, AA y Controles) no es igual o superior a 5 , se considerará que el estudiante no ha superado la asignatura. 


\subsection{Cronograma}

La satisfactoria utilización de las plataformas virtuales como apoyo a la docencia presencial requiere que el calendario de las actividades programadas en el aula virtual se imbrique con el de las actividadas a realizer en en aula física, dotando, además, de cierta flexibilidad en la timing de los entregables. A modo de ejemplo se plantea el siguiente cronograma en el que destaca, por un lado, que la apertura de los contenidos teórico prácticos de un modulo debe coincidir con el inicio de cada semana, de modo que el alumno pueda organizar su calendario ateniéndose al orden prescrito en la asignatura, y, por otro, que en determinadas ocasiones, la exigencia lectiva de la actividad puede exigir que la entrega de las actividades evaluables se prolongue más allá de la duración seminal asignada a cada módulo.

\section{Tabla 12. Cronograma}

\begin{tabular}{|c|c|c|c|c|}
\hline Actividad & $\begin{array}{c}\text { Tiempo estimado de } \\
\text { dedicación }\end{array}$ & Fecha inicio & $\begin{array}{l}\text { Fecha } \\
\text { fin }\end{array}$ & № Días \\
\hline Inicio del curso módulo 0 & $1 / 2 \mathrm{~h}$ & Semana1 & Semana1 & 1 \\
\hline Módulo 1.- Aspectos básicos del Derecho Financiero & $4 \mathrm{~h}$ & Semana 1 & Semana 1 & 7 \\
\hline Estudio de la unidad didáctica & $2 \mathrm{~h}$ & Semana 1 & Semana 1 & 7 \\
\hline AA1 & $2 \mathrm{~h}$ & Semana 1 & Semana 1 & 7 \\
\hline Módulo 2.- El tributo & $4 \mathrm{~h}$ & Semana 2 & Semana 2 & 7 \\
\hline Estudio de la unidad didáctica & $2 \mathrm{~h}$ & Semana 3 & Semana 3 & 7 \\
\hline AEC1 & $2 \mathrm{~h}$ & Semana 3 & Semana 3 & 7 \\
\hline Módulo 3.- La relación jurídico tributaria. & $71 / 2 \mathrm{~h}$ & Semana 3 & Semana 6 & 21 \\
\hline Estudio de la unidad didáctica & $3 \mathrm{~h}$ & Semana 3 & Semana 3 & 7 \\
\hline $\mathrm{AA} 2$ & $3 \mathrm{~h}$ & Semana 3 & Semana3 & 7 \\
\hline Control & $3 / 2 \mathrm{~h}$ & Semana 3 & Semana 6 & 21 \\
\hline Módulo 4.- Procedimientos de aplicación de los tributos & $8 \mathrm{~h}$ & Semana 4 & Semana 6 & 14 \\
\hline Estudio de la unidad didáctica & $4 \mathrm{~h}$ & Semana 4 & Semana 4 & 7 \\
\hline AEC2 & $4 \mathrm{~h}$ & Semana 4 & Semana 6 & 14 \\
\hline Módulo 5.- Ilícitos tributarios y procedimiento sancionador. & $2 \mathrm{~h}$ & Semana 5 & Semana & 7 \\
\hline Estudio de la unidad didáctica & $1 \mathrm{~h}$ & Semana 5 & Semana 5 & 7 \\
\hline $\mathrm{AEC3}$ & $1 \mathrm{~h}$ & Semana 5 & Semana 5 & 7 \\
\hline Módulo 6.- Recursos y reclamaciones contra la Hacienda. & $5 \mathrm{~h}$ & Semana 6 & Semana 6 & 14 \\
\hline Estudio de la unidad didáctica & $2 \mathrm{~h}$ & Semana 6 & Semana 6 & 7 \\
\hline AA3 & $3 \mathrm{~h}$ & Semana 6 & Semana 7 & 14 \\
\hline Módulo 7.- Integración práctica de los módulos anteriores. & $71 / 2 h$ & Semana 7 & Semana 10 & 21 \\
\hline $\mathrm{AEC4}$ & $6 \mathrm{~h}$ & Semana 7 & Semana 10 & 21 \\
\hline Control & $1 / 2 \mathrm{~h}$ & Semana 7 & Semana 10 & 21 \\
\hline Notificación de acreditaciones & & & & 2 días \\
\hline Cierre del curso & & Semana 11 & Semana 11 & \\
\hline
\end{tabular}

\section{Resultados}

La iniciativa presentada se encuentra aún en fase de implantación, por lo que no es posible, todavía, determinar de forma empírica el alcance de los objetivos pretendidos. Ello no obstante, la experiencia de asignaturas afines, y sobre todo las inestimables enseñanzas proporcionadas por el Servicio de Capus Virtual de la UPV-EHU, permiten ser halagüeños en cuanto a los provechosos resultados que se vayan a obtener.

En este sentido, las calificaciones alcanzadas por los alumnos en las actividades evaluables realizadas en estas primeras semanas permiten ser positivos respecto a unos resultados que parece van a ser atrayentes.

\section{Conclusiones}

Si bien los resultados obtenidos en términos de objetivos de aprendizaje y competencias del alumnado recogidos en la fase de implantación conminan a calificar la experiencia como muy positiva, lo cierto es que el grado de implicación exigido a alumnos y profesorado puede, en ciertas ocasiones, tornar la balanza de esta valoración en sentido contrario. Por un lado, porque para que la iniciativa funciones es necesario coordinar la carga lectiva a la que se ven sometidos1@s alumn@s con el profesorado del resto de asignaturas que componen 
el curso en el que se imparte la disciplina, ya que, de no hacerlo, se pueden llegar a crear tensiones en el desarrollo académico de nuestr@s estudiantes. Por otro, porque si bien la preparación de los contenidos virtuales no dista del que es propio de los contenidos tradicionales (pues puede llegar a utilizarse el mismo repositorio, obviamente actualizado), la interactividad que se le supone a la plataforma virtual, incluso su ascendencia "social" (el mantenimiento de los foros, talleres, en su caso wikis...) requiere una ingente labor y dedicación por parte del docente en términos temporales y de esfuerzo didáctico. En este sentido, entendemos que la preparación de este tipo de entornos virtuales, que de suyo ocupa un tiempo mucho más prolongado que la clase presencial, debiera ser compensado o reconocido en la carga crediticia de los profesores encargados de la asignatura, al menos en los primeros cursos de implantación de la iniciativa.

Por su parte, aun estando todavía en una fase inicial en el desarrola del plan docente de lo que podríamos llamar una unidad formativa online como plataforma de apoyo a la enseñanza del Derecho Financiero y Tributario, lo cierto es que la experiencia acumulada, con sus aspectos positivos y negativos, puede ser, como en otras Universidades públicas de consolidada trayectoria presencial, de gran utilidad en la implantación de la docencia de esta disciplina para grupos de estudiantes que asisten a clase de forma totalmente virtual, o, por qué no, para la proyección de cursos más especializados en cursos abiertos al público no específicamente universitario a través de plataformas OCW o Mooc.

\section{Referencias}

AREA MOREIRA, A. (2018) "De la enseñanza presencial a la docencia digital. Autobiografía de una historia de vida docente", RED. Revista de Educación a Distancia, Núm. 56, https://www.um.es/ead/red/56/area.pdf [Consulta: 18 de febrero de 2019].

BAUTISTA, G. ; BORGES, F. ; FORÉS, A. (2006) "Didáctica universitaria en entornos virtuales de enseñanza-aprendizaje". Narcea: Madrid.

BORGES, F. (2005). "La frustración del estudiante en línea. Causas y acciones preventivas". Digithum, UOC. $\mathrm{N}^{\circ} 7$, http://www.uoc.edu/digithum/7/dt/esp/borges.pdf [Consulta: 19 de febrero de 2019]

MORENO GONZÁLEZ, S.; LUCHENA MOZO, G. (2014) «Formación e-learning en la enseñanza superior del Derecho: experiencia en la Universidad de Castilla-La Mancha», REDU Revista de Docencia Universitaria, Vol. 12 (3), $\mathrm{N}^{\circ}$ extraordinario, https://polipapers.upv.es/index.php/REDU/article/view/5501, [Consulta: 9 de febrero de 2019].

PRIETO, S. (2018) “Aprendizaje y tecnologías de la información y la comunicación”. Ed: CEF. Madrid. 